\title{
On a Characterization of the Haar Distribution on Compact Abelian Groups
}

\author{
I.P. Mazur \\ B. Verkin Institute for Low Temperature Physics and Engineering \\ National Academy of Sciences of Ukraine \\ 47 Lenin Ave., Kharkiv 61103, Ukraine \\ E-mail: mazurivan85@gmail.com
}

Received November 12, 2013

We prove a theorem which characterizes the Haar distribution on a compact Abelian group by the independence of $n$ linear forms of $n$ independent random variables. The coefficients of the forms are continuous endomorphisms of the group.

Key words: compact Abelian group, linear form.

Mathematics Subject Classification 2010: 39B99, 43A25, 60B15, 62E10.

\section{Introduction}

In the last years much attention has been paid to the generalization of the classical characterization theorems of mathematical statistic to various algebraic structures. The case when random variables take values in locally compact Abelian groups was studied in details (see, e.g., [1-8], [12-10]).

Let $X$ be a second countable compact Abelian group, $\xi_{i}, i=1,2, \ldots, n$, be independent random variables with values in $X$ and distributions $\mu_{j}$. Let $\alpha_{i j}, i, j=1,2, \ldots, n$ be continuous endomorphisms of the group $X$. Consider the linear forms $L_{j}=\sum_{i=1}^{n} \alpha_{i j} \xi_{i}, j=1,2, \ldots, n$.

The problem of the characterization of the Haar distribution on a compact Abelian group first was considered by J.H. Stapleton in [15]. He proved that if $X$ is a compact connected Abelian group, $\alpha_{i j}$ are integers and $\mu_{j}$ satisfy some conditions, then the independence of the linear forms $L_{j}$ implies that all $\mu_{j}$ are the Haar distributions on $X$.

In [8], G.M. Feldman studied the problem of the chracterization of convolutions of Gaussian and Haar distributions on an arbitrary locally compact Abelian \footnotetext{
2015

This research was supported in part by the grant Network of Mathematical Research 2013-
}

(c) I.P. Mazur, 2014 
group. He proved, in particular, that if $X$ is a compact Abelian group, $n=2$, $\alpha_{i j}$ are topological automorphisms of $X$ satisfying some conditions, and $\mu_{j}$ has a continuous density, then the independence of the linear forms $L_{j}$ implies that $\mu_{j}$ are the Haar distributions on $X$.

The present paper is devoted to the characterization of the Haar distribution on compact Abelian groups. The above mentioned results proved in [15] and [8] follow from our main theorem.

Let $X$ be a locally compact Abelian group. Let $Y=X^{*}$ be the character group of $X$. If $x \in X$ and $y \in Y$, then denote by $(x, y)$ the value of the character $y$ at the element $x$. Let $K$ be a subgroup of $X$. Denote by $A(Y, K)=\{y \in Y$ : $(x, y)=1$ for all $x \in K\}$ its annihilator. Denote by $\operatorname{End}(X)$ the set of continuous endomorphisms of $X$, and by $A u t(X)$ the group of the topological automorphisms of $X$. For each $\alpha \in \operatorname{End}(X)$ denote by $\widetilde{\alpha}$ the adjoint continues endomorphism of the group $Y$ defined by the formula $(x, \widetilde{\alpha} y)=(\alpha x, y)$ for all $x \in X, y \in Y$. The identity automorphism of the group denote by $I$.

Denote by $\mathrm{M}^{1}(X)$ the convolution semigroup of probability distributions on $X$. Let $\mu \in \mathrm{M}^{1}(X)$. Denote by $\sigma(\mu)$ the support of $\mu$. The characteristic function of the distribution $\mu$ is defined by the formula

$$
\widehat{\mu}(y)=\int_{X}(x, y) d \mu(y), y \in Y .
$$

For $\mu \in \mathrm{M}^{1}(X)$ define the distribution $\bar{\mu} \in \mathrm{M}^{1}(X)$ by the formula $\bar{\mu}(B)=\mu(-B)$ for all Borel sets of $X$. Then $\widehat{\bar{\mu}}(y)=\overline{\widehat{\mu}}(y)$. Put $F_{\mu}=\{y \in Y: \widehat{\mu}(y)=1\}$. Then $F_{\mu}$ is a closed subgroup of $Y, \sigma(\mu) \subset A\left(X, F_{\mu}\right)$, the function $\widehat{\mu}(y)$ is invariant with respect to $F_{\mu}$, i.e., $\widehat{\mu}(y+h)=\widehat{\mu}(y), y \in Y, h \in F_{\mu}$.

Denote by $m_{X}$ a Haar measure on $X$. If $X$ is a compact group, we assume that $m_{X} \in \mathrm{M}(X)$. In this case the characteristic function of $m_{X}$ is of the form

$$
\widehat{m}_{X}(y)= \begin{cases}1, & y=0 \\ 0, & y \neq 0\end{cases}
$$

\section{The Main Theorem}

To prove the main theorem we need some lemmas.

Lemma 2.1. ([10]) Let $X$ be a second countable locally compact Abelian group, $Y$ be its character group. Let $\xi_{i}, i=1,2, \ldots, n, n \geq 2$, be independent random variables with values in $X$ and distributions $\mu_{i}$. The linear forms $L_{j}=\sum_{i=1}^{n} \alpha_{i j} \xi_{i}, j=1,2, \ldots, n$, where $\alpha_{i j} \in \operatorname{End}(X)$, are independent if and 
only if the characteristic functions $\widehat{\mu}_{i}(y)$ satisfy the equation

$$
\prod_{i=1}^{n} \widehat{\mu}_{i}\left(\sum_{j=1}^{n} \widetilde{\alpha}_{i j} u_{j}\right)=\prod_{i=1}^{n} \prod_{j=1}^{n} \widehat{\mu}_{i}\left(\widetilde{\alpha}_{i j} u_{j}\right), \quad u_{j} \in Y .
$$

Let $\mu \in \mathrm{M}^{1}(X)$. Expand $\mu$ into the sum

$$
\mu=a \mu_{a c}+b \mu_{s}+c \mu_{d},
$$

where $a \geq 0, b \geq 0, c \geq 0, a+b+c=1, \mu_{a c}$ is an absolutely continuous distribution with respect to $m_{X}, \mu_{s}$ is a singular distribution, and $\mu_{d}$ is a discrete distribution. We say that $\mu$ has a non-trivial absolutely continuous component with respect to $m_{X}$ if $a>0$.

Lemma 2.2. Let $X$ be a compact Abelian group, $Y$ be its character group, $\mu \in \mathrm{M}(X)$. Assume that $\mu$ has a non-trivial absolutely continuous component with respect to the $m_{X}$ and its support is not contained in a coset of a proper subgroup of $X$. Then

$$
\sup _{y \in Y, y \neq 0}|\widehat{\mu}(y)|<1
$$

P r o o f. If $X$ is finite, then the statement of Lemma 2.2 is obvious. Suppose that $X$ is not finite. Then $Y$ is an infinite discrete Abelian group. First note that

$$
\limsup _{k \rightarrow \infty}\left|\widehat{\mu}\left(y_{k}\right)\right|<1
$$

for any sequence $\left\{y_{k}\right\}$ which tends to infinity as $k \rightarrow \infty$. Indeed, it follows from (3) that

$$
\widehat{\mu}(y)=a \widehat{\mu}_{a c}(y)+b \widehat{\mu}_{s}(y)+c \widehat{\mu}_{d}(y), \quad y \in Y .
$$

By the condition $a>0$, and taking into account that $\lim _{k \rightarrow \infty} \widehat{\mu}_{a c}\left(y_{k}\right)=0$, we get from (6) that

$$
\limsup _{k \rightarrow \infty}\left|\widehat{\mu}\left(y_{k}\right)\right| \leq b+c<1 .
$$

Assume that there is a point $y_{0} \in Y$ such that $\left|\widehat{\mu}\left(y_{0}\right)\right|=1$. Put $\nu=\mu * \bar{\mu}$. Then $\widehat{\nu}\left(y_{0}\right)=\left|\widehat{\mu}\left(y_{0}\right)\right|^{2}=1$, and hence $F_{\nu} \neq\{0\}$. Put $G=A\left(X, F_{\nu}\right)$ and note that $G \neq X$. We have $\sigma(\nu) \subset G$. This implies that $\sigma(\mu)$ is contained in a coset of $G$, which is contrary to the condition of the lemma. Thus,

$$
|\widehat{\mu}(y)|<1, y \in Y, y \neq 0 .
$$

Assume that $\sup _{y \neq 0}|\widehat{\mu}(y)|=1$. This means that there exists a sequence $\left\{y_{k}\right\}_{k=1}^{\infty} \subset Y$ such that $\left|\widehat{\mu}\left(y_{k}\right)\right| \rightarrow 1$ as $k \rightarrow \infty$. Note that $Y$ is a discrete group because $X$ is a compact. Taking this into the account, it follows from (7) that the sequence $\left\{y_{k}\right\}_{k=1}^{\infty}$ tends to infinity as $k \rightarrow \infty$, but this contradicts to (5). The lemma is proved. 
Consider the matrix $\alpha=\left\{\alpha_{i j}\right\}_{i, j=1}^{n}$, where $\alpha_{i j} \in \operatorname{End}(X)$. The matrix $\alpha$ defines a continuous endomorphism $X^{n} \rightarrow X^{n}$ by a natural way. We also denote this endomorphism by $\alpha$. The main result of the paper is the following theorem.

Theorem 2.3. Let $X$ be a second countable compact Abelian group, $\xi_{i}, i=$ $1,2, \ldots, n$, be independent random variables with values in $X$ and distributions $\mu_{i}$. Assume that $\mu_{i}$ satisfy the conditions:

(i) $\sigma\left(\mu_{i}\right)$ is not contained in a coset of a proper subgroup of $X, i=1,2, \ldots, n$;

(ii) $\mu_{i}$ has a non-trivial absolutely continuous component with respect to the $m_{X}, i=1,2, \ldots, n$.

Assume that the matrix $\alpha=\left\{\alpha_{i j}\right\}_{i, j=1}^{n}$, where $\alpha_{i j} \in \operatorname{End}(X)$, satisfies the conditions:

(iii) every column of the matrix $\alpha$ contains at least two endomorphisms, which are epimorphisms;

(iv) $\alpha$ is a topological automorphism of $X^{n}$.

Then the independence of the linear forms $L_{j}=\sum_{i=1}^{n} \alpha_{i j} \xi_{i}, j=1,2, \ldots, n$, implies that $\mu_{i}=m_{X}, i=1,2, \ldots, n$.

P r o o f. Note that without loss of generality we can suppose that $\widehat{\mu_{i}}(y) \geq 0$. Indeed, put $\nu_{i}=\mu_{i} * \bar{\mu}_{i}$. Obviously, the distributions $\nu_{i}, i=1,2, \ldots, n$, satisfy conditions $(i)$ and $(i i)$. Moreover, $\widehat{\nu}_{i}(y)=\left|\widehat{\mu}_{i}(y)\right|^{2} \geq 0, y \in Y$. If we prove that $\nu_{i}=m_{X}$, this implies that $\mu_{i}=m_{X}, i=1,2, \ldots, n$.

By Lemma 2.1 the characteristic functions $\widehat{\mu_{i}}(y)$ satisfy Eq. (2). Taking into account that $\widehat{\mu}_{i}(y) \geq 0$, we can reformulate condition $(i)$ in the equivalent form

$$
F_{\mu_{i}}=\{0\}, \quad i=1,2, \ldots, n .
$$

Note that $\alpha_{i j}$ are epimorphisms if and only if Ker $\widetilde{\alpha}_{i j}=0$. Hence, we can reformulate condition (iii) in the following equivalent form:

$(i i i)^{\prime}$ for any $j_{0}$ there are at least two indexes $i_{1}, i_{2}$ such that the equalities $\operatorname{Ker}\left(\widetilde{\alpha}_{i_{1} j_{0}}\right)=\{0\}, \operatorname{Ker}\left(\widetilde{\alpha}_{i_{2} j_{0}}\right)=\{0\}$ hold.

Note also that $\alpha \in \operatorname{Aut}\left(X^{n}\right)$ if and only if $\widetilde{\alpha} \in \operatorname{Aut}\left(Y^{n}\right)$. It is obvious that $\widetilde{\alpha}$ acts by the formula

$$
\widetilde{\alpha}\left(u_{1}, u_{2}, \ldots, u_{n}\right)=\left(\sum_{j=1}^{n} \widetilde{\alpha}_{1 j} u_{j}, \sum_{j=1}^{n} \widetilde{\alpha}_{2 j} u_{j}, \ldots, \sum_{j=1}^{n} \widetilde{\alpha}_{n j} u_{j}\right), u_{j} \in Y .
$$

Assume that not all $\widehat{\mu}_{i}(y)$ are equal to $\widehat{m}_{X}(y)$. Then there exist $\widetilde{y} \in Y, \widetilde{y} \neq 0$, and $l_{0}$ such that

$$
\widehat{\mu}_{l_{0}}(\widetilde{y}) \neq 0 .
$$

Put $\left(\widetilde{u}_{1}, \widetilde{u}_{2} \ldots, \widetilde{u}_{n}\right)=\widetilde{\alpha}^{-1}(\widetilde{y}, 0, \ldots, 0)$. It is obvious that there exists at least one element $\widetilde{u}_{j}$ such that $\widetilde{u}_{j} \neq 0$. It is easy to see that the case when $\widetilde{u}_{j_{0}} \neq 0, \widetilde{u}_{j}=$ 
$0, j \neq j_{0}$, for some $j_{0}$ is impossible. Indeed, in this case, $\widetilde{\alpha}\left(0,0, \ldots, \widetilde{u}_{j_{0}}, \ldots, 0\right)=$ $\left(\widetilde{\alpha}_{1 j_{0}} \widetilde{u}_{j_{0}}, \widetilde{\alpha}_{2 j_{0}} \widetilde{u}_{j_{0}}, \ldots, \widetilde{\alpha}_{n j_{0}} \widetilde{u}_{j_{0}}\right)=(\widetilde{y}, 0, \ldots, 0)$ contrary to condition $(i i i)^{\prime}$. Hence, $\widetilde{u}_{j_{1}} \neq 0, \widetilde{u}_{j_{2}} \neq 0$ for some $j_{1}$ and $j_{2}$. Substituting $u_{i}=\widetilde{u}_{i}$ in (2), we obtain

$$
\widehat{\mu}_{l_{0}}(\widetilde{y})=\prod_{i=1}^{n} \prod_{j=1}^{n} \widehat{\mu}_{i}\left(\widetilde{\alpha}_{i j} \widetilde{u}_{j}\right) .
$$

Taking into account the inequality

$$
0 \leq \widehat{\mu}_{i}(y) \leq 1, \quad i=1,2, \ldots, n,
$$

and condition $(\text { iii })^{\prime}$, we can infer from equality (11) that the inequality

$$
\widehat{\mu}_{l_{0}}(\widetilde{y}) \leq \widehat{\mu}_{i_{1}}\left(\widetilde{\alpha}_{i_{1} j_{1}} \widetilde{u}_{j_{1}}\right) \widehat{\mu}_{i_{1}}\left(\widetilde{\alpha}_{i_{1} j_{2}} \widetilde{u}_{j_{2}}\right) \widehat{\mu}_{i_{2}}\left(\widetilde{\alpha}_{i_{2} j_{1}} \widetilde{u}_{j_{1}}\right) \widehat{\mu}_{i_{2}}\left(\widetilde{\alpha}_{i_{2} j_{2}} \widetilde{u}_{j_{2}}\right)
$$

holds for some $i_{1}, i_{2}$. From $(13)$ and $\widehat{\mu}_{1}(\widetilde{y}) \neq 0$ it follows that

$$
\widehat{\mu}_{i}\left(\widetilde{\alpha}_{i j_{1}} \widetilde{u}_{j_{1}}\right) \neq 0, \widehat{\mu}_{i}\left(\widetilde{\alpha}_{i j_{2}} \widetilde{u}_{j_{2}}\right) \neq 0, i=i_{1}, i=i_{2},
$$

where $\widetilde{u}_{j_{1}} \neq 0, \widetilde{u}_{j_{2}} \neq 0$. Since $\widetilde{u}_{j_{1}} \neq 0, \widetilde{u}_{j_{2}} \neq 0$ and $\operatorname{Ker}\left(\widetilde{\alpha}_{i j_{1}}\right)=\{0\}, \operatorname{Ker}\left(\widetilde{\alpha}_{i j_{2}}\right)=$ $\{0\}$, we have that $\widetilde{\alpha}_{i j_{1}} \widetilde{u}_{j_{1}} \neq 0, \widetilde{\alpha}_{i j_{2}} \widetilde{u}_{j_{2}} \neq 0$. Thus, we proved the following statement.

If (10) holds, then

$$
\widehat{\mu}_{l_{0}}(\widetilde{y}) \leq \widehat{\mu}_{l_{1}}\left(\widetilde{y}_{l_{1}}\right) \widehat{\mu}_{l_{2}}\left(\widetilde{y}_{l_{2}}\right) \widehat{\mu}_{l_{3}}\left(\widetilde{y}_{l_{3}}\right) \widehat{\mu}_{l_{4}}\left(\widetilde{y}_{l_{4}}\right),
$$

where $\widetilde{y}_{l_{k}} \neq 0, k=1,2,3,4$. It follows from (15) that $\widehat{\mu}_{l_{k}}\left(\widetilde{y}_{l_{k}}\right) \neq 0, k=1,2,3,4$. Hence, in (15) we can replace $\widehat{\mu}_{l_{0}}(\widetilde{y})$ with $\widehat{\mu}_{l_{k}}\left(\widetilde{y}_{l_{k}}\right), k=1,2,3,4$. As a result, we get

$$
\widehat{\mu}_{l_{0}}(\widetilde{y}) \leq \prod_{k=1}^{16} \widehat{\mu}_{l_{k}}\left(\widetilde{y}_{l_{k}}\right)
$$

for some $\widetilde{y}_{k} \neq 0, k=1,2, \ldots, 16$. By repeating this reasoning, we obtain the inequality

$$
\widehat{\mu}_{l_{0}}(\widetilde{y}) \leq \prod_{k=1}^{4^{m+1}} \widehat{\mu}_{l_{k}}\left(y_{l_{k}}\right),
$$

where $y_{l_{k}} \neq 0, k=1,2, \ldots, 4^{m+1}$.

Put

$$
C_{i}=\sup _{y \neq 0} \widehat{\mu}_{i}(y) .
$$

By Lemma 2.2, we have that $C_{i}<1$. Put

$$
C=\max _{1 \leq i \leq n} C_{i}
$$


Then $C<1$. From (17),(18) and (19) it follows that

$$
\widehat{\mu}_{1}(\widetilde{y}) \leq C^{4^{m+1}} \text {. }
$$

Since $C^{4^{m+1}} \rightarrow 0$ as $m \rightarrow \infty$, we can infer that $\widehat{\mu}_{l_{0}}(\widetilde{y})=0$, which contradicts the assumption. Therefore $\widehat{\mu}_{i}(y)=0$ for all $y \in Y, y \neq 0$, i.e., $\widehat{\mu}_{i}(y)=\widehat{m}_{X}(y), y \in Y$. Hence $\mu_{i}=m_{X}, i=1,2, \ldots, n$.

\section{Remarks}

We are to prove that Theorem 2.3 fails if we omit one of conditions $(i)-(i v)$.

We need some new notations. Denote by $\mathbb{T}$ the circle group, by $\mathbb{Z}$ the group of integers. The group $\mathbb{Z}$ is the character group of $\mathbb{T}$. Denote by $\mathbb{Z}(m)$ the group of the roots of the power $m$ of the unity. Denote by $\mathbb{Q}$ the additive group of rational numbers with the discrete topology. For an arbitrary $\boldsymbol{a}=\left(a_{0}, a_{1}, a_{2}, \ldots\right), a_{j}>1$, consider the group

$$
\mathbb{H}_{\boldsymbol{a}}=\left\{\frac{m}{a_{0} a_{1} \ldots \ldots a_{n}}: m \in \mathbb{Z}, n=0,1,2 \ldots\right\}
$$

which is a subgroup of the group $\mathbb{Q}$. The character group of $\mathbb{H}_{\boldsymbol{a}}$ is called the $\boldsymbol{a}$-adic solenoid and is denoted by $\Sigma_{\boldsymbol{a}}$. The group $\Sigma_{\boldsymbol{a}}$ is compact and connected. Note that the character group of the group $\mathbb{Q}$ is $\Sigma_{\boldsymbol{a}}$, where $\boldsymbol{a}=(2,3,4, \ldots)$

We remind that a distribution $\gamma \in \mathrm{M}^{1}(X)$ is called Gaussian (see [13, Ch. IV]) if its characteristic function can be represented in the form

$$
\widehat{\gamma}(y)=(x, y) \exp \{-\varphi(y)\}, \quad y \in Y,
$$

where $x \in X$, and $\varphi(y)$ is a continuous nonnegative function on $Y$ satisfying the equation

$$
\varphi(u+v)+\varphi(u-v)=2[\varphi(u)+\varphi(v)], \quad u, v \in Y .
$$

Denote by $\Gamma(X)$ the set of Gaussian distributions on $X$. Denote by $I(X)$ the set of idempotent distributions on $X$, i.e., the set of shifts of the Haar distributions $m_{K}$ of compact subgroups $K$ of $X$. Let $x \in X$. Denote by $E_{x}$ the degenerate distribution concentrated at the point $x \in X$.

Now we will show that if one of condition $(i)-(i v)$ is not satisfied, then Theorem 2.3 fails.

1. Put $X=\Sigma_{\boldsymbol{a}} \times \mathbb{Z}(3), \boldsymbol{a}=(2,2,2, \ldots)$. Consider independent identically distributed random variables $\xi_{1}$ and $\xi_{2}$ with values in $X$ and distribution $\mu=m_{K}$, where $K=\Sigma_{\boldsymbol{a}}$. Consider the linear forms $L_{1}=\xi_{1}+\xi_{2}, L_{2}=\xi_{1}-\xi_{2}$. We have $Y \cong \mathbb{H}_{\boldsymbol{a}} \times \mathbb{Z}(3), \boldsymbol{a}=(2,2,2, \ldots)$. To avoid introducing new notations, suppose that $Y=\mathbb{H}_{\boldsymbol{a}} \times \mathbb{Z}(3)$. For an arbitrary group $G$, denote by $f_{2}$ the homomorphism 
$f_{2}: G \rightarrow G$ defined by the formula: $f_{2} g=2 g, g \in G$. It is obvious that $f_{2} \in \operatorname{Aut}(Y)$, and hence $f_{2} \in \operatorname{Aut}(X)$.

On the one hand, it is obvious that condition $(i)$ does not hold, but conditions (ii) $-($ iv) hold.

On the other hand, taking into account that $f_{2}(K)=K$ as noted in [4, Proposition 7.4], the linear forms $L_{1}$, and $L_{2}$ are independent, herewith $\mu \neq m_{X}$.

2. Put $X=\Sigma_{\boldsymbol{a}}, \boldsymbol{a}=(2,3,4, \ldots)$. As in item 1 , we assume that $Y=\mathbb{Q}$. Let $\mu \in \Gamma(X)$ and $\widehat{\mu}(y)=\exp \left\{-y^{2}\right\}$. It is obvious that $\{y \in Y:|\widehat{\mu}(y)|=1\}=\{0\}$. Hence $(i)$ holds.

Since $X$ is a connected and not locally connected group, the distribution $\mu$ is singular ([4, Proposition 3.14]). Hence (ii) is not fulfilled.

Consider independent identically distributed random variables $\xi_{1}$ and $\xi_{2}$ with values in the group $X$ and distribution $\mu$. Consider the linear forms $L_{1}=\xi_{1}+\xi_{2}$ and $L_{2}=\xi_{1}-\xi_{2}$. It is obvious that conditions (iii) and (iv) hold.

It follows from Lemma 2.1 that the linear forms $L_{1}$ and $L_{2}$ are independent, herewith $\mu \neq m_{X}$.

3. Let $X$ be a finite Abelian group such that $X$ is not isomorphic to the group of the form $\mathbb{Z}(p)$, where $p$ is a prime number. It was proved (see [10, Proposition $3.2]$ ) that there exist independent identically distributed random variables $\xi_{1}$ and $\xi_{2}$ with values in $X$ and distribution $\mu$ and nonzero endomorphisms $\beta, \delta$ of the group $X$ such that the following conditions hold:

(a) the linear forms $L_{1}=\beta \xi_{1}+\delta \xi_{2}$ and $L_{2}=\xi_{1}+\beta \xi_{2}$ are independent;

(b) $\mu \notin I(X)$;

(c) $\sigma(\mu)=X$;

(d) $\beta^{2}-\delta \in \operatorname{Aut}(X)$;

(e) $\beta \notin \operatorname{Aut}(X)$.

Condition $(i)$ follows from $(c)$. Condition $(i i)$ is fulfilled because we consider the group $X$ in the discrete topology. It follows from $(e)$ that condition (iii) does not hold. Finally, $(d)$ implies $(i v)$.

4. Put $X=\Sigma_{\boldsymbol{a}}, \boldsymbol{a}=(3,3,3, \ldots)$. Consider the function

$$
\rho(x)=1+\operatorname{Re}\left(x, y_{0}\right),
$$

where $x \in X, y_{0} \in Y, y_{0} \neq 0$. It is obvious that $\rho(x) \geq 0, x \in X$, and $\int_{X} \rho(x) d m_{X}(x)=1$. Let $\mu$ be a distribution on $X$ with the density $\rho(x)$ with respect to $m_{X}$. Then $\sigma(\mu)=X$. Let $\xi_{i}, i=1,2,3$, be independent identically distributed random variables with values in $X$ and with distribution $\mu$. Consider the linear forms $L_{1}=\xi_{1}+\xi_{2}+\xi_{3}, L_{2}=\xi_{1}+3 \xi_{2}+\xi_{3}, L_{3}=\xi_{1}+\xi_{2}+3 \xi_{3}$. Obviously, conditions $(i)-(i i i)$ hold. Moreover, it is easy to see that $\alpha=\left(\begin{array}{lll}1 & 1 & 1 \\ 1 & 3 & 1 \\ 1 & 1 & 3\end{array}\right) \notin$ 
Aut $\left(X^{3}\right)$ because $(0,1,0) \notin \widetilde{\alpha}\left(Y^{3}\right)$, i.e., (iv) does not hold. On the other hand, as proved in [11], the linear forms $L_{1}=\xi_{1}+\xi_{2}+\xi_{3}, L_{2}=\xi_{1}+3 \xi_{2}+\xi_{3}, L_{3}=$ $\xi_{1}+\xi_{2}+3 \xi_{3}$ are independent.

\section{References}

[1] G.M. Feldman, More on the Skitovich-Darmois Theorem for Finite Abelian Groups. - Theory Probab. Appl. 45 (2000), 507-511.

[2] G.M. Feldman, On a Characterization Theorem for Locally Compact Abelian Groups. - Probab. Theory Relat. Fields 133 (2003), 345-357.

[3] G.M Feldman and P. Graczyk, On the Skitovich-Darmois Theorem for Discrete Abelian Groups. - Theory Probab. Appl. 49 (2005), 527-531.

[4] G.M. Feldman, Functional Equations and Characterization Problems on Locally Compact Abelian Groups. EMS Tracts in Mathematics 5, Zurich, European Mathematical Society, 2008.

[5] G.M. Feldman, Independent Iinear Statistics on a-Adic Solenoids. - Theory Probab. Appl. 54 (2010), 375-388.

[6] G.M. Feldman and P. Graczyk, The Skitovich-Darmois Theorem for Locally Compact Abelian Groups. - Australian Math. Soc. 88 (2010), 339-352.

[7] G.M. Feldman and M.V. Myronyuk, Independent Linear Forms on Connected Abelian Groups. - Mathematische Nachrichten 284 (2011), 255-265.

[8] G.M. Feldman, On a Characterization of Convolutions of Gaussian and Haar Distributions. - Mathematische Nachrichten 286 (2013), 340-348.

[9] E. Hewitt and K.A. Ross, Abstract Harmonic Analysis. Vol. 1. Springer-Verlag, Berlin, Gottingen, Heildelberg, 1963.

[10] I. Mazur, The Skitovich-Darmois Theorem for Finite Abelian Groups (the case of $n$ linear forms of $n$ random variables). - Ukr. Math. J. 4 (2011), 1512-1523.

[11] I. Mazur, On the Skitovich-Darmois Theorem for a-Adic Solenoids. arXiv:1303.4238, 2013.

[12] M.V. Myronyuk and G.M. Feldman, Independent Linear Statistics on the TwoDimensional Torus. - Theory Probab. Appl. 52 (2008), 78-92.

[13] K.R. Parthasarathy, Probability Measures on Metric Spaces. Chelsea Publishing (AMS), 1967.

[14] K. Schmidt, On a Characterization of Certain Infinitely Divisible Positive Definite Functions and Measures. - J. London Math. Soc. 4 (1972), 401-407.

[15] J.H. Stapleton, A Characterization of the Uniform Distribution on a Compact Topological Group. - Ann. Math. Stat. 34 (1963), 319-326. 